\title{
INSTITUCIONES
}

\section{APLICACIÓN DE LA LITERATURA ALEMANA EN LOS ESTUDIOS UNIVERSITARIOS DE DAF EN ALEMANIA. BREVE INFORME PARA PROFESORES, ESTUDIANTES E INVESTIGADORES.}

$\mathrm{H}$ ace ya casi cuatro años que la mayoría de las universidades andaluzas y españolas implantaron los nuevos planes de estudios viéndose abocadas necesariamente a reajustes curriculares y metodológicos para los que, en muchos casos, no se habia actuado con la suficiente previsión. Esta puede ser también, de hecho, la circunstancia de las segundas lenguas y sus literaturas, en que más que "reajustes" se ha tenido que llevar a cabo un diseño casi completamente nuevo de la materia a impartir. La disminución considerable del creditaje asignado ahora a las antiguas segundas lenguas ha de soportar, además, el obligado reparto entre contenidos lingüisticos y literarios ${ }^{1}$. Cómo esto se viene haciendo en los últimos cuatro años es una cuestión de máxima relevancia para quienes, como nosotros, aceptaran el reto de diseñar el ensamblaje de los nuevos contenidos sin renunciar, por ello, a los principios metodológicos que habian regido su labor docente hasta ese momento. Tanto es asi, que, lejos de abandonar las pautas didácticas de adquisición que, en nuestro caso, habian presidido los primeros pasos de la relación con la lengua, la inclusión de contenidos literarios en nuestros programas supuso la reaplicación o inserción de dichos contenidos en nuestro propio modus docendi con unas cotas de éxito impensables antes de la experimentación. Lógicamente, el desarrollo de dichas pautas didácticas no ha sido consecuencia del azar y sí, como puede adivinarse, de una intensa investigación sobre los contenidos curriculares en si, el momento de su introducción propiamente dicha, o la forma en que habian de presentarse, $y$, todo ello, convenientemente justificado y razonado en base a los principios metodológicos por que nos regimos?

La reciente y creciente reavivación de los aspectos referidos a la interculturalidad por las editoriales dedicadas al diseño de manuales para la ensenanza del alemán es otra de las razones que apoyan con más firmeza la inclusión de la literatura en la realidad de las lenguas extranjeras ${ }^{3}$. $Y$ es que la literatura puede verdaderamente constituir la manifestación artistica que, mediante un instrumental lingüistico, presente la mayor carga de información explicita o implícita sobre patrones culturales propios y/o ajenos. No obstante, los elementos implicados en el proceso de enseñanza-aprendizaje que los diseñadores de estos $u$ otros textos no siempre tienen en consideración son demasiados; y las necesidades, los modos y las vias de renovación, por otro lado, son tan particulares y diversas, que, más que una solución general -que diera por bueno el que todas las lineas investigadoras atendieran a las mismas directrices didácticas-, se hace necesaria una especie de "ideario" a partir del que poder tomar contacto con 
el desarrollo del tema en cuestión, quizás una guia comentada de los centros docentes e investigadores que de este tema se ocupan.

A continuación ofreceremos un breve paseo por el panorama docente e investigador de las universidades alemanas, algo asi como un informe práctico con el que abrir puertas a los docentes, dicentes e investigadores de este campo. Las universidades

\section{Augsburg}

En la Universidad de Augsburg se imparte lo que han dado en llamar "Deutsche Philologie unter besonderer Berücksichtigung des Deutschen als Zweitsprache und der Didaktik des Deutschen als Zweitsprache", una especialidad en la que, al margen de la lógica diferenciación que entre "segunda lengua" y "lengua extranjera" habriamos de hacers, no se

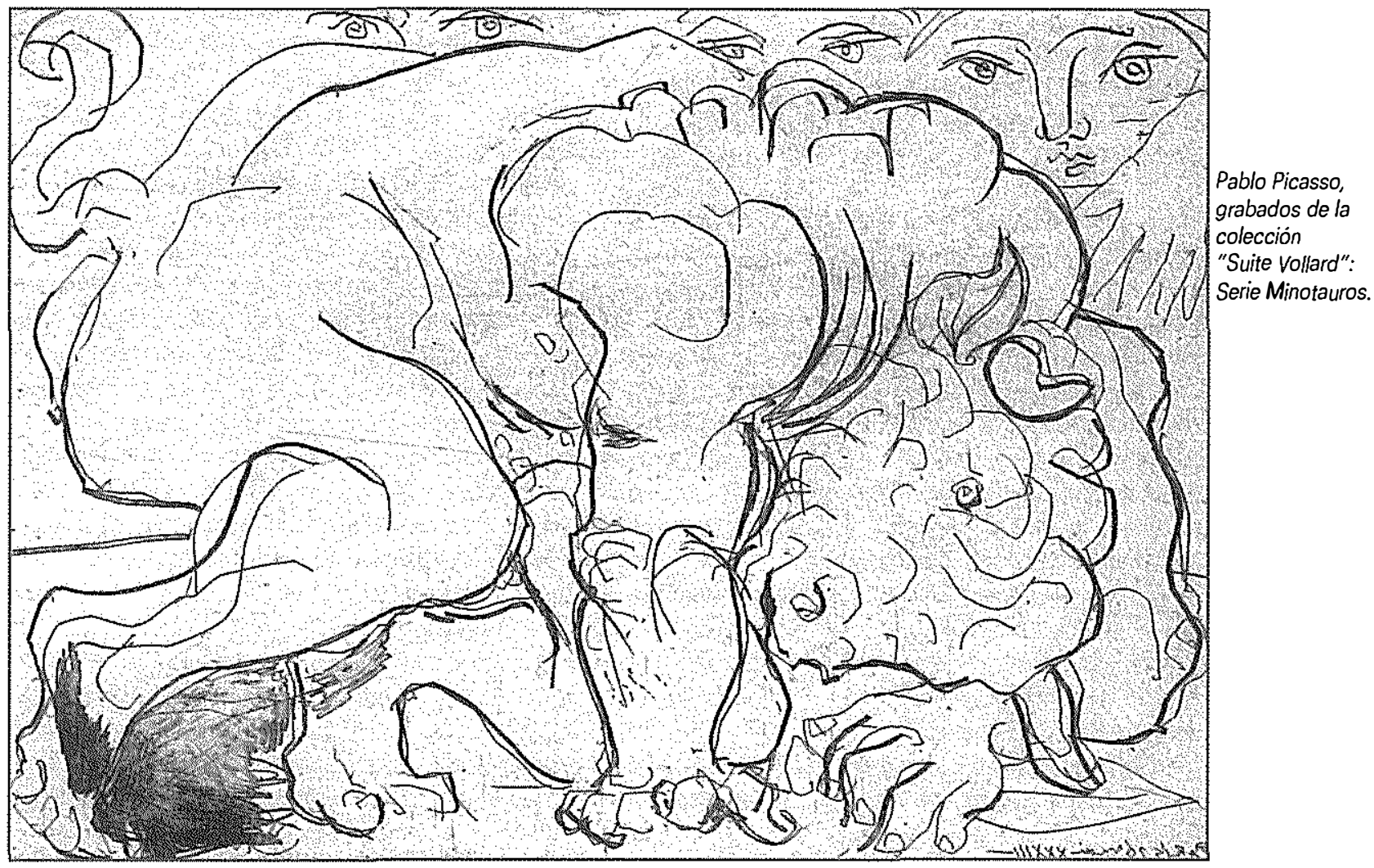

citadas no son todas las que comprenden estudios de $\mathrm{DaF}$, sino sólo aquéllas que subjetivamente hemos seleccionado bajo el criterio de la mayor o menor atención que, desde sus guias docentes $\mathrm{e}$ investigadoras, parecieran prestar al tema de la aplicación de la literatura a la ensenanza del alemán como lengua extranjera. La guia en cuestión tratará de resumir de forma clara la dedicación especifica de cada universidad al tema, comentando los seminarios que suelen ofertar ${ }^{4} y$ reseñando los proyectos referidos a la investigación de la aplicación de la literatura a la enseñanza de la lengua en que alguna de ellas pudiera estar embarcada. han dejado de buscar aplicaciones prácticas de la literatura en lengua alemana, asi como de la nueva comprensión de "interculturalidad" 6 , para la enseñanza del alemán. Durante el semestre de invierno del año en curso se imparten de hecho dos interesantes seminarios a cargo del Dr. Alev Tekinay. El primero de ellos trata del concepto "Fremde" y su significado en el campo del alemán como lengua extranjera para con la cultura, el llamado "Landeskunde", $y$, como no podia faltar, la literatura. La preceptiva justificación que en la correspondiente guia universitaria aparece, alude al papel de trascendental importancia que un concepto como "Fremde"

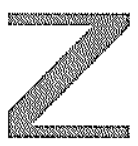


desempeña en el proceso de construcción de la actual sociedad multicultural $-y$, sobre todo, de cara a una convivencia harmónica entre nativos y extranjeros-, dejando ver también su reflejo en el campo del alemán como lengua extranjera. Es por ello que el seminario propone el tratamiento detallado de este concepto, analizando su significado lingüistico, la relación entre su contenido y la realidad, asi como su función cultural. El rostro práctico de dicho seminario habrá que buscarlo en las reflexiones y propuestas didácticas para el "Landeskunde" y la literatura, propuestas que habrán de conducir no sólo al terreno de lo puramente "escolar", sino también al de las relaciones sociolingüisticas e interculturales con los extranjeros alli implicados.

El segundo de los seminarios seleccionados en esta universidad lleva por titulo "Komparatistik am Beispiel der Frauenliteratur. Deutschsprachige Migrantinnenliteratur im Vergleich mit der Literatur muttersprachlicher Autorinnen: Sprachlicher Stil, Themen und Tendenzen". En él viene a reivindicarse que, del mismo modo que la lingüistica comparativa (o contrastiva) o que la cultura comparada suponen aspectos importantes de la especialidad de $\mathrm{DaF}$, no podemos dejar de conceder el mismo grado de importancia a la comparación entre literaturas, no ya de las llamadas literaturas nacionales, sino de otros tipos "menores" de literatura perteneciente a lo que denominan "Frauenliteratur", literatura femenina (hecha por mujeres). $Y$ es que, si dicha literatura está por regla general desatendida como tal, mucho menos eco tienen las obras de mujeres escritoras que, desde su calidad de inmigrantes, han empezado en los últimos años a escribir en alemán. Las cuestiones de partida giran en torno al dominio que éstas tienen de la lengua alemana, o bien, los temas sobre los que escriben estas mujeres, e, incluso, las tendencias que muestran en sus obras. Pero lo verdaderamente interesante para nosotros es que, al margen de la comparación con la producción literaria de autoras nativas, y además de permitir una visión general de la literatura femenina actual en lengua alemana, el seminario propone la investigación sobre las posibilidades de su utilización didáctica en la clase de alemán como lengua extranjera.

\section{Bielefeld}

La especialidad de "Deutsch als Fremdsprache" de la Universidad de Bielefeld cuenta también entre sus docentes con especialistas en el campo de la "Literatur im DaF-Unterricht" (literatura en la enseñanza del alemán como lengua extranjera), como por ejemplo el Dr. Lutz Köster, quien, sin embargo no imparte actualmente seminarios sobre esta disciplina de los que tengamos noticias. Lo que quizás resulte más relevante de esta especialidad es el proyecto de investigación que hace unos anos (19901993) se llevó a cabo en esta misma universidad. El proyecto en cuestión, que titularian "Entwicklung von Materialien für einen integrierten fachsprachlichen und literatursprachlichen DaF-Unterricht", tenia por objetivo la preparación de materiales didácticos de orientación especifica al tipo de discente con que se tratara, es decir, atendiendo primordialmente a los objetivos prácticos que, en cada caso, tuvieran los alumnos de un determinado grupo. Los materiales en cuestión serian fundamentalmente textos que apoyaran, por una parte, la integración laboral, $y$, por otra, la comprensión de patrones culturales y sociales ajenos, distintos a los propios. Los textos didactizados pertenecerian al mundo empresarial o puramente literario, y estarian clasificados según criterios temáticos. Todos ellos serian después utilizados en seminarios de la especialidad de Ciencias Empresariales dirigidos a estudiantes extranjeros, comprobando in situ su verdadera utilidad y aprovechamiento. Los resultados fueron enormemente positivos y se puede acceder a los textos en el $\mathrm{DaF}-\mathrm{CaF}$ de esta misma universidad. No obstante, y como en el caso de Augsburg, la investigación parece haber estado dirigida al proceso de enseñanza-aprendizaje de la lengua alemana en una situación de "segunda lengua", por lo que, si bien puede suponer un punto de partida para investigaciones de este tipo en nuestro pais, seria absolutamente necesario corregir también aqui el planteamiento inicial para adecuarlo a una situación de "lengua extranjera" propiamente dicha.

\section{Bochum}

En la Universidad de Bochum se ofertan estudios complementarios (Zusatzstudiengang) de verdadero interés desde nuestro planteamiento de partida, y es que en dicha universidad la especialidad si separa lo que denomina "Deutsch als Zweitsprache" de la que viene presentada como "Deutsch als Fremdsprache / Deutschunterricht im Ausland", unos estu-

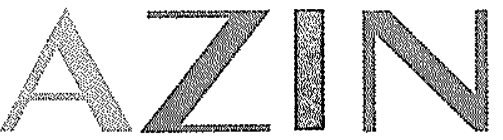


dios organizados en seis campos de conocimiento que abarcan tanto la lingüística, como el "Landeskunde", pasando por el que denominan "Deutsche Literatur aus eigener und fremder Perspektive". Junto a ello, los estudios de cada especialidad filológica proponen cursos destinados a la didáctica de la literatura de una lengua extranjera, de modo que, además de seminarios inicialmente neutrales -como el titulado "Textarbeit im Fremdsprachenunterricht" del profesor Krings-, pertenecientes a la especialidad de "Didáctica de la lengua extranjera" (Studiengang Sprachlehrforschung), los estudiantes pueden acceder a otros cursos de carácter más específico: tal es el caso, por ejemplo, del que los docentes Behrens y Kahr propusieran el pasado semestre sobre "Die Vermittlung von Lektüretechniken für literarische Texte auf der Sekundarstufe I und II" (dirigido a estudiantes de Magisterio de las especialidades de inglés, francés, italiano, ruso y espanol). No menos interesantes resultan los cursos de "Didáctica de la Literatura" que, en el terreno del inglés como lengua extranjera, imparte el profesor Mosner en esta universidad: tanto el primero de ellos, titulado "Teaching R. C. Sheriff's Journey's End to advanced learners of English, como el segundo, "Talking books in the literature class", invitan a la investigación empirica propia, llevando a cabo la práctica real de las propuestas metodológicas que se hagan.

\section{Dresden}

La Universidad Técnica de Dresde también oferta asignaturas de "Germanistica-Alemán como lengua extranjera" (Germanistik-DaF) como materias troncales o secundarias de algunas licenciaturas. Entre los contenidos de dichas materias encontramos también los referidos al bloque de "Literatura intercultural", cursos estos que versan sobre literatura moderna alemana, literatura de viajes, o, quizás más interesante para nosotros, sobre "la imágen de lo ajeno en la literatura alemana". Estos cursos del ciclo básico entroncan con otros del ciclo principal en los que se ven contenidos sobre la imagen propia (de Alemania) en la literatura universal, la literatura universal infantil y juvenil, o la literatura de emigración. Pero entre lo más interesante y reciente queremos destacar dos de los seminarios que se imparten: de un lado el que dirige el Dr. H. Rösch sobre "Literaturpädagogik und Migrationsliteratur", en que el segundo concepto sirve como corpus de contenido teórico para la pedagogia de la literatura; y, por otra parte, el más generalmente titulado "Literatur im $\mathrm{FU}(\mathrm{DaF})^{\prime \prime}$, que imparte $Z$. Parpulova, uno que toca de lleno las cuestiones más importantes de la aplicación de la literatura a la enseñanza del alemán como lengua extranjera -aunque, visto el panorama, hayamos de dudar sobre el verdadero enmarcamiento del seminario: $\mathrm{DaF}$ o DaZ-.

\section{Greifswald}

También en la Enrst-Moritz-Arndt-Universität de Greifswald se imparten estudios de alemán como lengua extranjera asociados a una especialidad de "Germanistica para extranjeros". Entre los cursos y seminarios del ciclo básico hemos querido rescatar aqui algunos que nos pueden resultar interesantes. Comenzemos con el que imparte Angela Bader

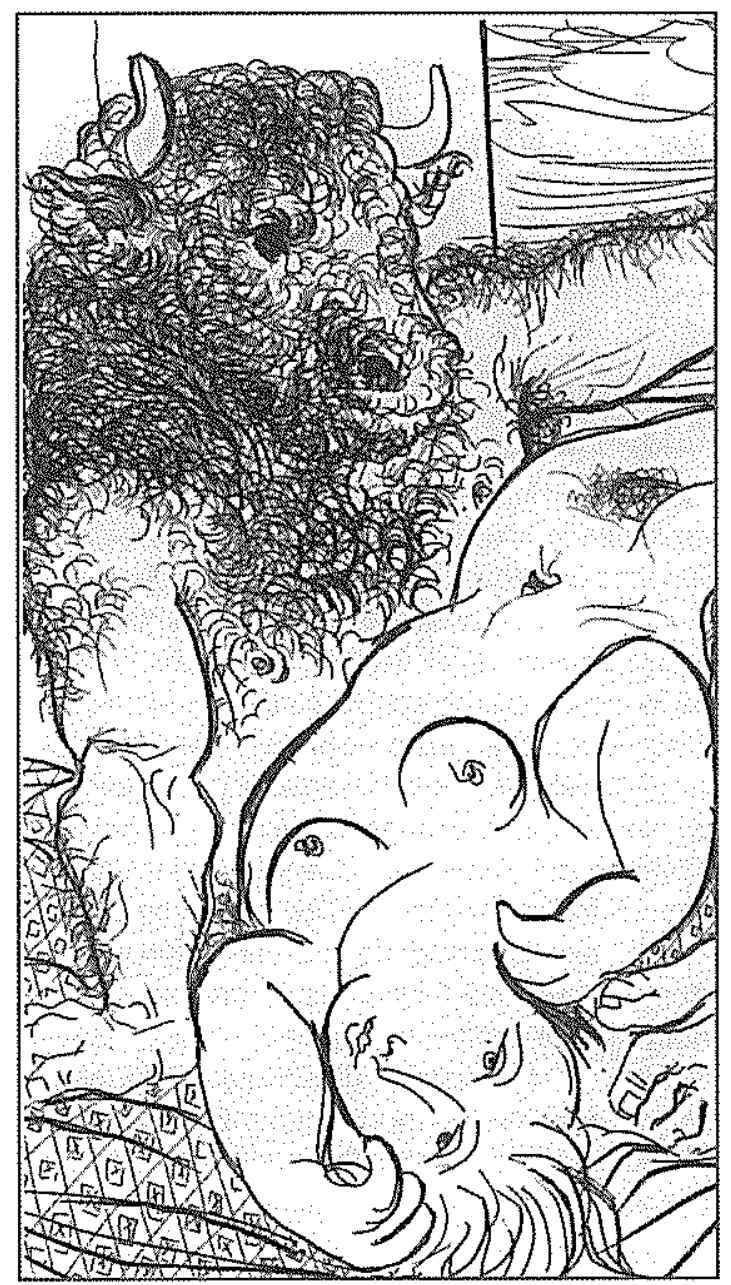

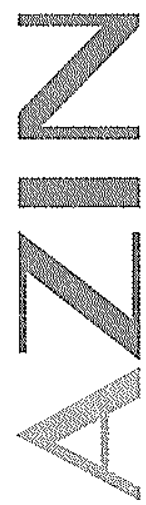


sobre "Landeskunde Deutschlands aktuell", un seminario en el que tienen cabida una selección de temas muy actuales en el panorama social alemán: "política y organización educativa", "problemas de la juventud en la Alemania del Este tras la anexión", "juventud y violencia", "tendencias de extrema derecha", "relaciones Iglesia-Estado en la Alemania actual", "influencias de las sectas en el pais", etc. Todos estos temas pueden ser analizados también en relación con acontecimientos históricos que

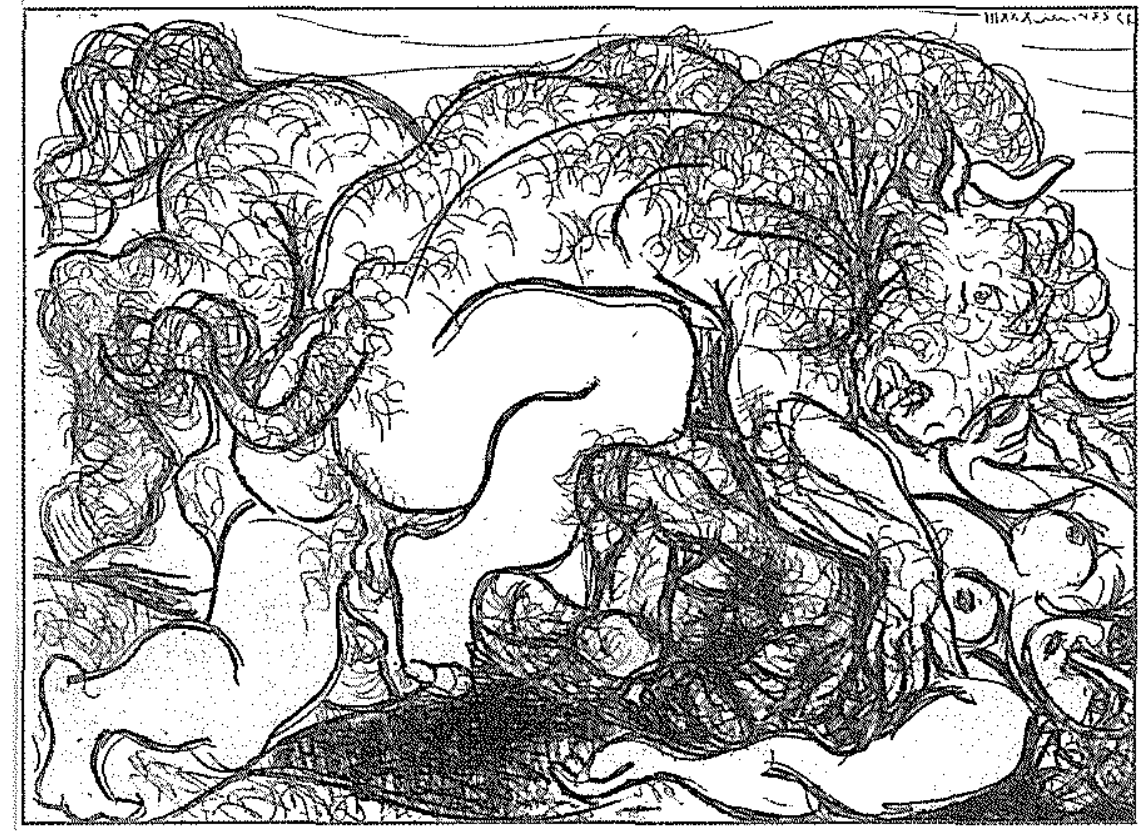

expliquen la situación actual, buscando referentes literarios escritos $u$ orales que pudieran representar la raiz de los problemas de nuestros días. Esta misma docente nos introduce de lleno en el terreno de la lengua escrita con un curso que imparte con Gisela Ros sobre "Texto y estrategias textuales en la clase de lengua y en la clase de lengua extranjera", un planteamiento contrastivo de alto interés para la didáctica del alemán como lengua extranjera en el que se tratan diversas teorías modernas sobre el "texto" bajo las claves de su clasificación y tipificación. Los criterios y métodos de análisis y sintesis que aquí se ponen en práctica prestan especial atención a las estrategias didácticas a desarrollar en una situación específica de lengua extranjera, y proponen fórmulas concretas tanto para textos del campo de la técnica como para textos poéticos y literarios en general.
Pero, para la verdadera utilización de textos literarios en la enseñanza del alemán como lengua extranjera habremos de asistir al seminario de introducción que Karl-Heinz Borchardt propone bajo el título de "Literarische Texte im Fach Deutsch als Fremdsprache". Aqui se pretenden extraer conclusiones del análisis de la aplicación de la literatura a la enseñanza de la lengua atendiendo también a la cultura ajena que, por medio de aquélla, se presenta. Junto a esto se investiga en la selección de un corpus literario adecuado y se llevan a cabo reflexiones sobre el modo de utilización de estos textos en situaciones de "lengua extranjera". El mismo docente imparte otro seminario de carácter más específico y, si cabe, práctico sobre la utilización del "Hörspiel" en la clase de alemán como lengua extranjera, um módulo didáctico en el que, partiendo de la aportación de Annette Berndt (1994)7, se buscan formas de didacticar piezas tradicionales $y$ modernas de esta particular manifestación literaria. Es también Borchardt quien en el ciclo básico de la Germanistica para Extranjeros oferta un seminario titulado "Epische Kurzformen und Möglichkeiten ihres Einsatzes im Fach Deutsch als Fremdsprache", centrado fundamentalmente en la "Kurzgeschichte" de posguerra, aunque también se tratan otras formas literarias breves de la prosa moderna. El valor del seminario una vez más radica, como su título expresa, en la búsqueda de fórmulas válidas para la aplicación de un tipo de literatura a la enseñanza de la lengua. Ya en el ciclo principal propone este docente otro seminario de caracteristicas metodológicas similares, pero centrado ahora en las adaptaciones cinematográficas de piezas literarias. Borchardt parte aqui no sólo de la percepción y valoración de la realidad y de la obra literaria a través de su adaptación al cine, sino, y sobre todo, de las posibilidades de aplicación didáctica de estas obras para una explotación productiva de la lengua meta. Esto to hace sin renunciar a la actual y constante valoración contrastiva de la obra literaria y la cinematográfica, y expone las posibilidades de aprovechamiento de la forma de recepción literaria más extendida en el mundo. Si en el seminario anterior el único apoyo bibliográfico lo constituian las recopilaciones y valoraciones criticas de las obras que formaban el corpus, es decir, sin material específico sobre su aplicación didáctica a la enseñanza del alemán como lengua extranjera, ahora cuenta el autor con dos estudios de interés, que, bien tratados, 
pueden significar al menos una forma metodológica de importancia: el de Brande y Helmling (1985) 8 sobre la utilización de la cámara en la enseñanza del alemán como lengua extranjera, por un lado, y el de Hurst $(1995)^{9}$, por otra parte, sobre el análisis comparativo de obras literarias y sus adaptaciones cinematográficas.

\section{Halle}

En la Martin-Luther-Universität de Halle-Wittenberg se ofertan asignaturas del "Area de Alemán como Léngua Extranjera" para complementar la especialidad de Lingüistica Germánica, en la que apenas hay una atención verdadera a la aplicación de la literatura a la enseñanza de la lengua. Si se imparten sin embargo dos cursos que, al menos, rozan el núcleo de interés aqui evaluado: el primeo de ellos, bajo el título de "Literatura alemana como literatura extranjera", profundiza en la imagen de Alemania que la literatura alemana exporta a otros espacios no germanófilos, buscando las claves emocionales y cognitivas de dichos espacios para la percepción y valoración que hagan del objeto representado (Alemania) y del medio por el que lo representan (la literatura), al tiempo que se extraen conclusiones sobre sus posibilidades de aplicación didáctica a la enseñanza del alemán como lengua extranjera; el segundo si se concentra ya de forma más práctica en la producción y evaluación de textos en la clase de lengua extranjera ("Texte schreiben und bewerten im FSU") partiendo del clásico análisis sobre las diferencias entre lengua escrita y lengua oral en manuales, libros de texto, pero deja de lado desde su fundamento la utilización de textos literarios.

\section{Hamburg}

Los estudios parciales de Germanistica que aqui se imparten para estudiantes extranjeros parten del campo de la "Investigación en Didáctica de la Lengua" (Sprachlehrforschung), con sede en el Instituto Central de Lenguas Extranjeras de esta universidad. Aqui, junto a especialidades de inglés, francés, ruso o español, se oferta "Deutsch als Fremdsprache", estructurado en tres campos que comprenden: el dominio práctico del idioma, la enseñanzaaprendizaje de las lenguas, y la práctica docente propiamente dicha. Esta estructuración se justifica mediante la concepción inicial de la "investigación didáctica" que las reune, una investigación que ellos gustan definir como "centrada en el aprendiente, empirica e interdisciplinar o integrativa". Contempla efectivamente los elementos afectivos y cognitivos del discente, tanto individuales como globales, al tiempo que proponen hipótesis y teorias didácticas propias extraidas de la experimentación directa en la realidad de la didáctica de la lengua extranjera aunque no especifican si tampoco aqui distinguen entre situaciones de DaF y DaZ-, y todo ello sin

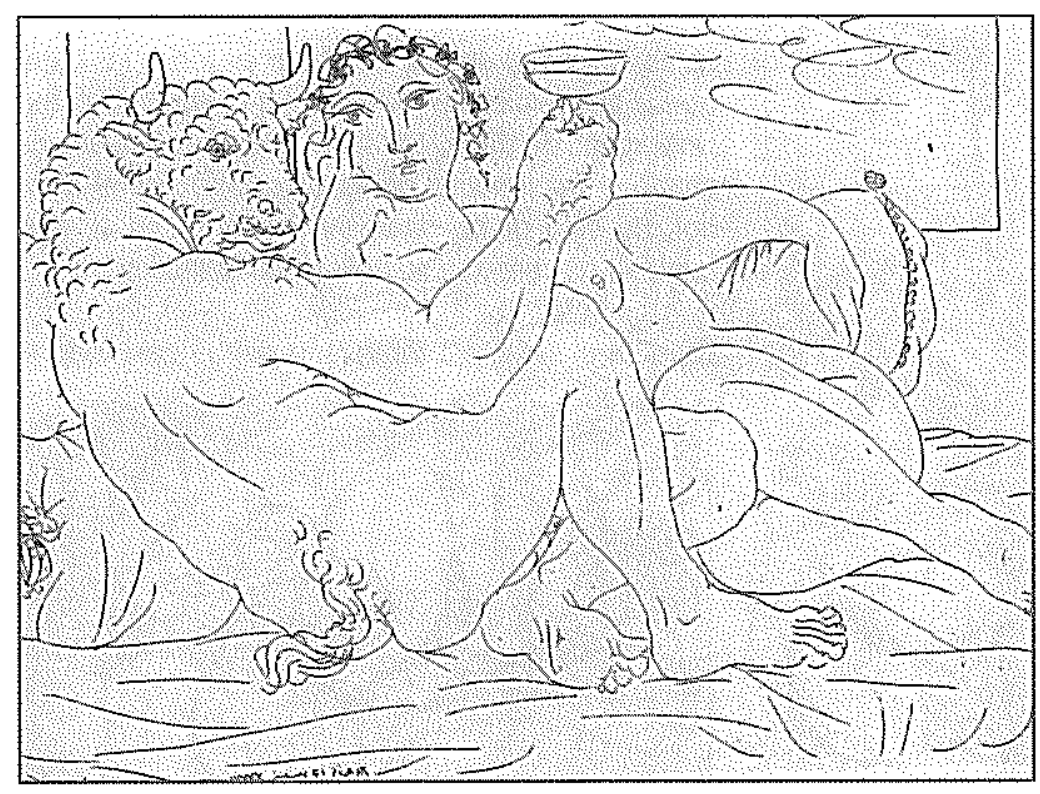

dejar de buscar referentes teóricos y prácticos en otros terrenos más o menos ajenos a la didáctica de la lengua, entre los que rescatamos ya, junto a los de la sociologia, psicologia, lingüistica aplicada, etc, el referido a la literatura. Tanto es asi que en los últimos años se han venido desarrollando diferentes proyectos de investigación cuyos desarrollos $y$ resultados pueden suponer un modelo o un punto de partida para investigaciones más concretas sobre la aplicabilidad de la literatura a la enseñanza del alemán como lengua extranjera en Andalucia. Börner ha dirigido el primero de ellos, entre 1990 y 1993,sobre el análisis didáctico del uso escrito de la lengua en la enseñanza de la lengua extranjera, un proyecto que complementa el dirigido por Edmondson apenas dos anos antes sobre la literatura en la didáctica de la lengua extranjera, asi como el más específico de Montua sobre la integración de un determinado tipo de literatura en la enseñanza de

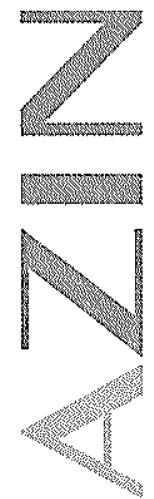


lenguas. Al margen de los consabidos problemas de especificación de las situaciones de lengua extranjera o de segunda lengua que se dan tanto en el terreno de la docencia como en el de la investigación, los diferentes proyectos sólo contemplan, en cualquier caso, integraciones literarias en niveles más o menos intermedios y avanzados que no limitan excesivamente los condicionamientos lingüisticos del texto ni de la interacción o el discurso didáctico.

\section{Jena}

La especialidad de DaF se oferta en todas las categorias académicas posibles en la Friedrich-SchillerUniversität de Jena, tratando la literatura alemana desde la perspectiva de su percepción en el exterior, no sólo como disciplina científica en sí, sino también en cuanto a su aplicación didáctica.

Durante el semestre de verano del 98 casi todo el

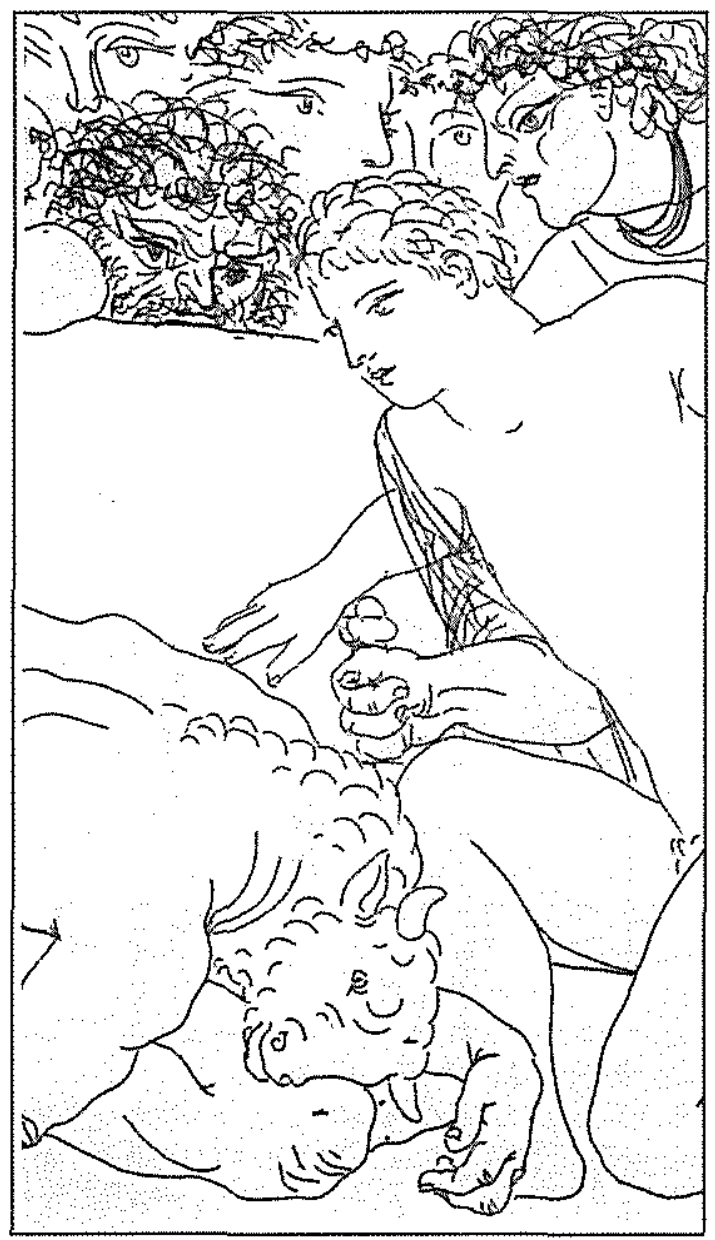

peso de la docencia dedicada a la literatura en la enseñanza del alemán como lengua extranjera vuelve a recaer en el Dr. Werner Biechele, quien organiza varios seminarios en que se integran los trabajos de investigación literaria en el terreno del DaF. Este es el caso, sin ir más lejos, del seminario que bajo el titulo de "Einführung in die Literaturinterpretation" propone en el ciclo básico, un seminario fundamentado en la comprensión de la recepción literaria como proceso comunicativo y que parte de las experiencias lectoras individuales ${ }^{10}$ tratadas desde los patrones de la estética de la recepción. Su contenido abarca el propio análisis literario, rescatando, como base para la enseñanza de la lengua, la terminologia específica de la literatura y la propia práctica de la investigación literaria en si misma. También se plantean otras cuestiones de interés en cuanto a la recepción y transmisión de la literatura alemana en el extranjero, un terreno que conduce indiscutiblemente al segundo de los seminarios propuestos por Biechele, precisamente al que titula "Didaktik des Fremdverstehens", un curso que se apoya en la idea, a nuestro parecer muy acertada, de que la "comprensión" depende no tanto del perfecto dominio de un determinado sistema lingüistico, como del conocimiento de las convenciones y patrones culturales que en cada caso se manifiesten junto al mensaje lingüistico. El seminario propone textos de diferentes disciplinas (medicina, psicologia, filosofia o derecho) que tematizan diversos aspectos relacionados con la interculturalidad directamente, pero culmina con el tratamiento de estos en obras literarias europeas y alemanas. Plantea para ello preguntas clave sobre la medida en que lo ajeno condiciona el aprendizaje de la lengua extranjera, sobre lo que significa verdaderamente la comprensión de lo ajeno y la forma en que esta comprensión puede ser enseñada o aprendida en la clase, o sobre las posibilidades de la enseñanza reglada para conseguir que los estudiantes realmente logren en mayor o menor medida adoptar la perspectiva desde la que los ajenos (extranjeros) en cuestión perciben la realidad.

También en el ciclo básico propone otro seminario sobre el tratamiento hermenéutico de los textos literarios en la clase de lengua extranjera ${ }^{11}$ en el que también apuesta por la interculturalidad desde el subtitulo "Wege zur interkulturellen Kommunikation" que lo define. El seminario trata de definir el tipo de 
preguntas sobre un texto literario con el que se puede desarrollar una clase de lengua extranjera ${ }^{12}$. El Dr. Biechele pretende comprobar el gran potencial estimulador que guardan los textos de ficción para los aprendientes de la lengua, asi como las posibilidades de experimentación con textos auténticos en el proceso de aprendizaje. Las propuestas didácticas resultantes del seminario están dirigidas a confirmar el verdadero papel de los metodólogos, es decir, la tarea de diseñar materiales pensados para la interacción entre el texto y el lector con los que se logre ampliar los conocimientos culturales, estéticos y lingüisticos del aprendiente. No menos importantes para él resultan los criterios bajo los que se determinan los objetivos didácticos y, más importante aún, los textos a tratar en clase, textos literarios de paises germanófonos, o de literaturas extranjeras en lengua alemana, 0 , incluso, de autores extranjeros a la literatura una especial relevancia cultural que, de ningún modo, puede ser despreciada por omisión en la clase de lengua extranjera. La literatura, como la concibe este docente, consigue hacer de lo ajeno algo susceptible de ser vivido en la experiencia propia de lo cotidiano, con lo que sensibiliza al lector para con los otros modos de vida y conduce a una percepción diferente de la realidad. Estas ideas conectan con el segundo de los seminarios que sobre el particular de la aplicación de la literatura a la enseñanza del alemán como lengua extranjera imparte Biechele en el ciclo principal, nos referimos al titulado "Deutschsprachige Literatur des Auslands und ihr Beitrag zum Dialog der Kulturen". Aqui, lógicamente, la orientación es prioritariamente hacia la conexión que sin lugar a dudas existe entre la literatura y la interculturalidad. Para ello se plantea un ligero análisis contrastivo entre las literaturas en

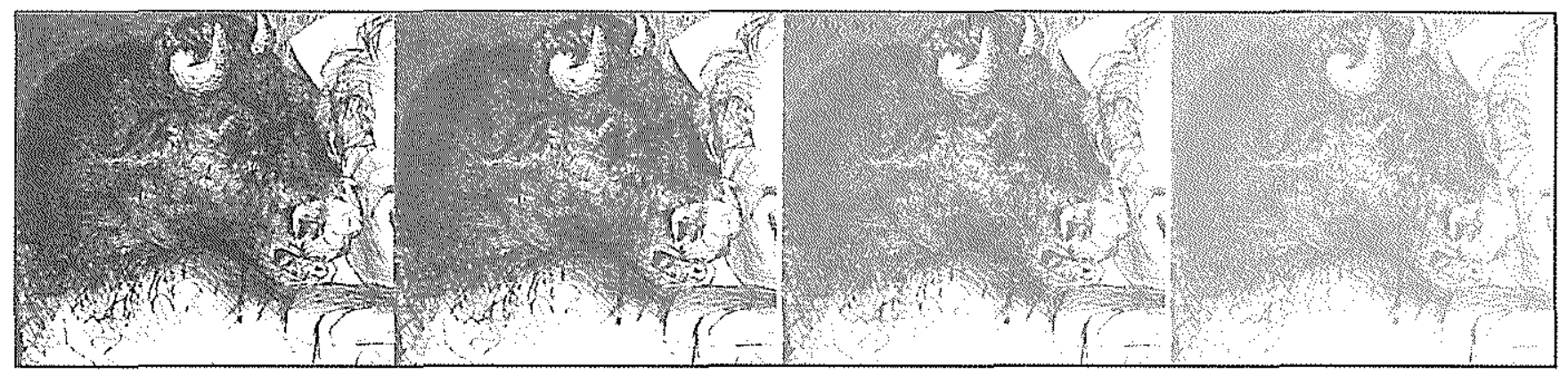

que escriban en alemán, textos que, en definitiva, resulten especialmente apropiados para el aprendizaje de patrones interculturales en una clase de lengua extranjera programada por tareas.

En el ciclo principal la oferta didáctica del profesor Biechele se hace más concreta y práctica aún, con un seminario en el que se revisa la literatura alemana posterior a 1945 desde una perspectiva histórico-social, o sea, analizándo las coyunturas sociales y políticas de la época, las vidas y circunstancias particulares de los autores, asi como sus posicionamientos ideológicos, aspectos todos estos implicitos en un planteamiento hermenéutico de la aplicación de la literatura a la clase de lengua extranjera. Las cuestiones sobre la función de la literatura son refundadas aqui, asi como las diferentes percepciones de los procesos evolutivos de la sociedad que cada autor manifiesta en sus obras. Lo fundamental es que estos aspectos histórico-sociales conceden lengua alemana producidas en Europa Occidental, Europa Oriental y Norteamérica, que contribuya al conocimiento básico necesario para desarrollar tareas docentes como lector de alemán en estas zonas del mundo. El corpus literario con que trabaja recoge textos de F. Kafka, P. Celan, R. Ausländer, H. Müller, F. Hodjak, V. Koch, G. Niers y A. Weckmann, entre otros, que se analizan desde el punto de visa político-cultural, critico-literario, y de otros aspectos implicados en su recepción en la República Federal de Alemania. Este seminario se inscribe en un proyecto común con universidades americanas para la elaboración de un volumen de lectura de literatura alemana en norteamérica, exportable a otras zonas de afluencia de autores alemanes en la historia moderna.

Por último quisieramos hacer referencia al proyecto "DaF-Dossier Weimar" que durante el semestre de invierno ha estado a cargo del Dr. Rainer Bettermann 
y de Regina Werner. El proyecto en cuestión conecta los conceptos de "literatura" y "Landeskunde" bajo la idea, presentar, discutir y elaborar una especie de "bolsa de material" para DaF en que se pueda apreciar, fundamentalmente a través de textos literarios, la percepción alemana de lo propio y lo ajeno.

\section{Leipzig}

El área de "InterDaF/Herder-Institut" de la Universidad de Leipzig oferta a los estudiantes de Germanistica cursos y seminarios sobre la didáctica del alemán, de la literatura alemana y del "Landeskunde" en los paises germanófonos en general. Margit Ebersbach imparte dentro del campo de conocimientos obligatorio "Didáctica de la literatura" el curso titulado "Deutschprachige Literatur im DaF-Unterricht unter literaturdidaktischen Gesichtspunkten". Aqui, la multiplicidad de mensajes y de emociones de los textos literarios proporcionan una magnífica ocasión para la expresión libre, el hablar sin limitaciones sobre los modos de comprender una cultura ajena. Los textos utilizados por Ebersbach pertenecen a la prosa breve y la lírica de nuestro siglo, y exigen el continuo mejoramiento por la práctica de las competencias comunicativa y cultural. Son textos sobre los que se aplican estrategias lectoras prácticas para la lengua extranjera y fórmulas de recepción y análisis de los contenidos, si bien también se tratan los criterios de selección que conducen a los propios textos y se analizan los elementos que los hacen susceptibles de ser aplicados en los diferentes niveles de la enseñanza de la lengua.

El curso que esta misma docente imparte sobre "Literarische Erkundungen des Fremden: Fremde und Fremdes in der Literatur" analiza el conocimien- to que se tiene sobre otras culturas o sobre el propio concepto de "fremd" y su significado en la comunicación intercultural que la literatura en general propone. Para ello se revisa parte de la literatura alemana en que se hace manifiesto el concepto, ya sean libros de viaje que aún hoy influyen en la imagen que tenemos del exterior u otro tipo de obras que tematizen igualmente una particular recepción de lo ajeno'13.

\section{Marburg}

El Área de Alemán com Lengua Extranjera de la Universidad de Marburg plantea la circunstancia metodológica particular de centrar sus seminarios didácticos en la ensenanza de adultos, tanto en Alemania, como en el extranjero. Junto a esta circunstancia, las Dras. Ruth Albert y Anthonya Visser, que dirigen esta especialidad, se reparten los contenidos lingüisticos y literarios respectivamente para concentrarse en sus posibilidades didácticas en el campo de DaF. A. Visser es quien se encarga principalmente del terreno de la literatura, con seminarios sobre la recepción (transmisión) de la literatura en lengua alemana, diversos conceptos asociados al "Landeskunde" y su didáctica, la aplicación en general a la enseñanza del alemán como lengua extranjera, el desarrollo de materiales didácticos en general, o la interculturalidad en la enseñanza-aprendizaje de la lengua extranjera. Lo más importante, a nuestro entender, es aqui el reconocimiento de circunstancias diferentes para la enseñanza del alemán en Alemania y fuera de ella, asi como la especialización en aprendientes adultos, aunque tampoco aqui se especifica si la aplicación que de los materiales se haga a la práctica docente se refiere a niveles iniciales, medios o avanzados en cuanto al dominio del idioma.

\section{München}

El departamento de "Alemán como Lengua Extranjera" de esta universidad ha propuesto en el recién terminado semestre de invierno varios seminarios especificamente dirigidos a la aplicación de la literatura en DaF. Entre ellos queremos subrayar los dos que tratan de forma especifica las destrezas escritas. El que bajo el titulo de "Lesen und Lesedidaktik im Bereich DaF" impartiera el Prof. Esselborn se refiere exclusivamente a los elementos propios de la 
recepción escrita, partiendo de una revisión histórica desde el siglo XVIII en que toma cuerpo el sistema literario (en el más amplio sentido del término: publicación, mercado, bibliotecas, etc) hasta nuestros dias (en que se introducen estudios de crítica literario-sociológica). El tratamiento de la lectura en la enseñanza de la lengua extranjera toma como punto de partida el concepto de "Verstehensdidaktik" (Neuner) que se impusiera en los anos 8014 y trata de desarrollar posibilidades investigadoras contrastivas entre el proceso lector con textos en la lengua materna y en lengua extranjera, análisis que quieren culminar con la presentación de materiales didácticos especificos para la comprensión lectora global y detallada de textos en lengua extranjera: estrategias (como ayudas para la lectura-comprensión) que puedan presentarse como sistemáticas para una metodología específica del tratamiento de textos literarios en la clase de alemán.

El Prof. Graefen complementa la propuesta de Esselborn con su seminario sobre "Schrift und Schreiben im Unterricht Deutsch als Fremdsprache", en el que analiza, como expresa el título, la destreza productiva de la lengua escrita. En este seminario se analizan las implicaciones de la lengua escrita para la adquisición de la lengua en general, asi como la necesidad de una mayor especificidad metodológica para el proceso de enseñanza de adultos. Se ofrecen, en consecuencia, nuevos modelos didácticos de desarrollo de la actividad escritora a partir de la observación comparativa de casos de monolingüismo y plurilingüismo, tanto con ninos, como con adultos. Se trata de desarrollar propuestas concretas que, por una parte, logren paliar los problemas especificos de la ortografia, $y$, por otra, portencien $y$ mejoren la competencia productiva textual en todas sus facetas.

Pero este autor hace también una valoración general de gran interés para la didáctica de la lengua escrita en su seminario " "Text" als linguistische Grundkategorie - "Texte« als Unterrichtsmaterial". Aqui se analiza (constata o critica) el valor de los textos escritos para la didáctica de los idiomas, al tiempo que se proponen determinados textos desde su autenticidad- por su proximidad a lo cotidiano y su contribución al "Landeskunde". Los problemas que aqui se denuncian y tratan son de corte exclusivamente didáctico y metodológico, por lo que se revisan las contribuciones teóricas sobre la utilización de este tipo de material en la clase de alemán como lengua extranjera.

La valoración que a modo de conclusión queremos aportar ha de subrayar la dificultad de reconocer en las correspondientes guias universitarias el verdadero sentido de los conceptos que utilizan. La distinción entre $\mathrm{DaF}$ y $\mathrm{DaZ}$ no es rigurosa en casi ninguno de los casos comentados, hecho que, entre otras razones, se desprende del apoyo bibliográfico que en la mayoria de las universidades proponen ${ }^{15}$. Este hecho plantea ya un cierto tono de incertidumbre sobre la posibilidad verdadera de servirnos de estas esperiencias docentes e investigadoras como referencia para las propias, a lo que aún hemos de sumar la falta de especificidad en cuanto a los objetivos que en cada caso plantean: los cursos en que se reconocen contenidos literarios en un entorno académico de DaF suelen proponer, en apariencia, objetivos puramente literarios, aspirando, en el mejor de los casos, a seleccionar un corpus adecuado y a diseñar un modelo didáctico para la transmisión de dichos contenidos, pero abandonan la idea de proponer objetivos lingüisticos paralelos o implicitos en los modelos metodológicos de sus clases de literatura. Por último quisiéramos también destacar el hecho innegable de que, aunque las propuestas didácticas se centran en diseños destinados a aprendientes adultos (universitarios) o adolescentes (para los casos de Enseñanza Secundaria), en ningún caso se refieren a aprendientes de nivel inicial, dando por hecho, entendemos que prematuramente, la imposibilidad de aplicar los materiales y contenidos literarios a la enseñanza del alemán como lengua extranjera con alumnos sin conocimientos previos. Todo ello, conviene insistir, apunta a la necesidad de adaptar todas las sugerencias didácticas a nuestra más inmediata $y$, permitaseme, "cruda" realidad.

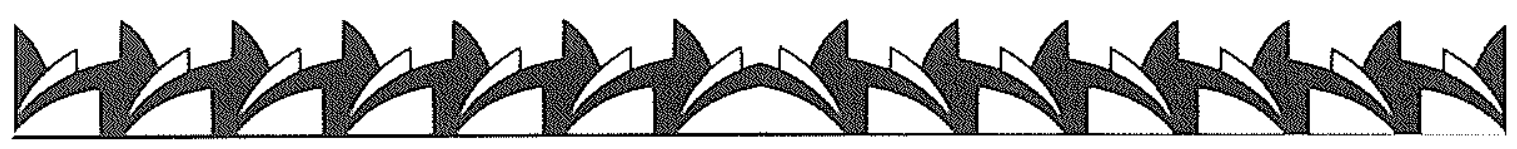




\section{NOTAS}

1 La situación es denunciada desde sus inicios por los investigadores y docentes que en la adaptación de sus materias a la nueva organización curricular advierten el grave empeoramiento de las condiciones de aprendizaje. Cf. Haidl, A. (1996): "„DaF in Andalusien«. Überlegungen und Hypothesen zu einer möglichen Arbeitsrichtung". En Magazin, 1 (1996), págs. 16-32, aqui pág. 17.

2 Una clara idea de dichos principios la ofrece Anton Haidl en su trabajo anteriormente citado " DaF in Andalusien«. Überlegungen und Hypothesen zu einer möglichen Arbeitsrichtung" (1996).

3 Las aportaciones más interesantes a nuestro parecer sobre el modo en que esto se lleve a efecto las hace en nuestro entorno el Prof. Manuel Maldonado Beltrán, en los trabajos que vino presentando en los primeros números de Magazin. Podemos recomendar el articulo "La literatura en la enseñanza del alemán como lengua extranjera" que escribe con Julia M. Borreguero Beltrán en Magazin, 1 (1996, págs. 8-15), asi como su segunda contribución, titulada "Constructivismo radical y enseñanza de la literatura", en Magazin, 2 (1997, págs. 35-41).

4 Téngase en cuenta que este informe ha sido elaborado a partir de los "proseminarios", los seminarios y los cursos que en cada centro se vienen impartiendo a lo largo de este curso académico (97/98), es decir, a partir de las noticias que hemos podido recoger de los correspondientes "Vorlesungsverzeichnisse". Ello quiere decir, que, en algunos casos, la materia ha sido impartida durante el pasado semestre de invierno, y, en otros, el curso está aún llevándose a cabo durante el presente semestre de verano. Por otra parte, hemos de advertir que la celebración de un determinado curso durante alguno de los semestres no implica necesariamente que se celebre también durante el mismo semestre del próximo curso académico, y si, en cambio, que en dicha universidad existe al menos la disposición a atender al tema en cuestión de que se trate en el curso.

5 Lamentablemente hemos de reconocer una vez más la falta de rigurosidad en la utilización indiscriminada de "segunda lengua" y "lengua extranjera" manifiesta en los resúmenes comentados de la mayoria de las universidades (de ésta, sin lugar a dudas) para referirse a una misma situación de aprendizaje (en este caso, fundamentalmente la de "segunda lengua"), como si de meros recursos dialécticos se tratara.

6 Precisamente la que se desprende de manuales como el Sichtwechsel 2. Mittelstufe Deutsch als Fremdsprache (Verlag Klett Edition Deutsch; München, 1996), en el que los contrastes conductuales y, con ello, conceptuales que son presentados se reflejan en el uso lingüistico. El propio manual incluye un área especifica de "Kulturvergleich" (págs. 48-85) con su correspondencia en el libro de ejercicios. Obsérvese como ejemplo el apartado de "Interkulturelles Lernen" de cada uno de los 10 temas que alberga este mismo manual.

7 Berndt, Annette(1994): Produktiver Einsatz von neuen Hörspielen und auditiver Dichtung im Unterricht Deutsch als Fremdsprache. München.

8 Brande, M.-L. u. B. Helmling (1985): Arbeit mit Video am Beispiel von Spielfilmen. Projekt Didaktik und Methodik für den Unterricht Deutsch als Fremdsprache. München.

9 Hurst, M. (1995): Erzählsituationen in Literatur und Film. Ein Modell zur vergleichenden Analyse von literarischen Texten und filmischen Adaptionen. Tübingen.

10 Son diferencias no exclusivas de los individuos, sino también de los grupos sociales a que estos pertenecen, como las que se proponen en "Leseunterschiede. Zum interkulturellen Lesegespräch", una contribución de trascendencia en: Jahrbuch Deutsch als Fremdsprache 7/1981.

11 Un planteamiento similar al que propone Hans Hunfeld (1990) en: Literatur als Sprachlehre, Ansätze eines hermeneutisch orientierten Fremdsprachenunterrichts. Tambiën la profesora Margit Ebersbach plantea esta perspectiva en sus cursos de la Universidad de Leipzig, comentados en este mismo trabajo.

12 Recomendamos sobre esta cuestión revisar las obras de Swantje Ehlers Literarische Texte lesen lernen (München, 1992) y/o Lesen als Verstehen (Berlin, 1992).

13 Sin duda alguna se ha basado para el planteamiento de este curso en el autor que posiblemente más atención ha dedicado a este tema, Dietrich Krusche en sus obras: "Fremde als Metapher. Beispiel aus der deutschen Lyrik seit der Jahrhundertwende" (en Jahrbuch DaF 8; Heidelberg, 1982), Literatur und Fremde (München, 1985), Reisen: Verabredung mit der Fremde (Berlin, 1989), o la más reciente Kafka in Japan, Goethe am Äquator, Deutsche Literatur im Ausland (Bonn, 1993). Sobre el tema en particular ha aparecido también una obra editada por Dietrich Harth titulada Fiktion des Fremden. Erkundung kultureller Grenzen in Literatur und Publizistik (Frankfurt am Main, 1994) que también recoge aspectos temáticos de interés para su aplicación a la enseñanza de la lengua alemana por medio de la literatura.

14 Cf. Karcher, Günther (1989): Das Lesen in der Erst- und Fremdsprache, Heidelberg, y Westhoff, Gerard J. (1987): Didaktik des Leseverstehens, München.

15 La gran mayoria de los comentarios que recogimos señalan algún tipo de bibliografia inicial en la que nunca faltan autores que, desde nuestro punto de vista, obvian la verdadera y necesaria distinción entre situaciones de aprendizaje de DaF y de DaZ, como por ejemplo los tan comunmente utilizados manuales de Bausch, R./ Christ, H./ Krumm, H.-J. (Handbuch Fremdsprachenunterricht. Francke: Tübingen/Basel 1995), o Rösler, D. (Deutsch als Fremdsprache. Metzler: Stuttgart, Weimar 1994). 